\title{
Aligned Graphene Nanoribbons and Crossbars from Unzipped Carbon Nanotubes
}

\author{
Liying Jiao', Li Zhang ${ }^{1}$, Lei Ding ${ }^{2}$, Jie Liu², and Hongjie Dai ${ }^{1}(\bowtie)$ \\ ${ }^{1}$ Department of Chemistry and Laboratory for Advanced Materials, Stanford University, Stanford, California 94305, USA \\ ${ }^{2}$ Department of Chemistry, Duke University, Durham, North Carolina 27708, USA \\ Received: 24 March 2010 / Revised: 30 March 2010 / Accepted: 31 March 2010 \\ (C) The Author(s) 2010. This article is published with open access at Springerlink.com
}

\begin{abstract}
Aligned graphene nanoribbon (GNR) arrays have been made by unzipping of aligned single-walled and few-walled carbon nanotube (CNT) arrays. Nanotube unzipping was achieved by a polymer-protected Ar plasma etching method, and the resulting nanoribbon array can be transferred onto any chosen substrate. Atomic force microscope (AFM) imaging and Raman mapping on the same CNTs before and after unzipping confirmed that $\sim 80 \%$ of CNTs were opened up to form single layer sub-10 nm GNRs. Electrical devices made from the GNRs (after annealing in $\mathrm{H}_{2}$ at high temperature) showed on/off current $\left(I_{\text {on }} / I_{\text {off }}\right)$ ratios up to $10^{3}$ at room temperature, suggesting the semiconducting nature of the narrow GNRs. Novel GNR-GNR and GNR-CNT crossbars were fabricated by transferring GNR arrays across GNR and CNT arrays, respectively. The production of such ordered graphene nanoribbon architectures may allow for large scale integration of GNRs into nanoelectronics or optoelectronics.
\end{abstract}

\section{KEYWORDS}

Graphene nanoribbon, single-walled carbon nanotubes, aligned, unzipping, plasma etching

Graphene nanoribbons have emerged as an interesting material with a wealth of electronic and spin transport properties [1-6]. Narrow (sub-10 nm) GNRs open up band gaps useful for field effect transistors (FETs) [1-4]. Several approaches have been developed to make GNRs, such as lithographic patterning [5-9], sonochemical methods $[1,10]$, chemical vapor deposition (CVD) $[11,12]$, nanocutting [13-15] and unzipping of carbon nanotubes (CNTs) [16-22]. For integration of GNRs into devices, in addition to controlling their width, edge smoothness and quality, it is important to control the alignment of GNRs. Among existing methods, lithographic patterning is capable of fabricating large arrays of aligned GNRs but the width and edge smoothness of GNRs are not well controlled, especially at the sub-10 nm scale. Unzipping CNTs offers a possibility to meet these challenges since much progress has been made on controlled synthesis (alignment, density, length and diameter controls) of CNTs in the past two decades [23-25]. Our previous work demonstrated that GNRs with well controlled width in the 10-20 $\mathrm{nm}$ range and smooth edges could be made by unzipping multiwalled carbon nanotubes (MWNTs) using a polymer-protected plasma etching method [16]. Most recently, we developed a new method that produced highly pristine nanoribbons

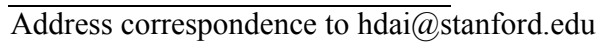


by unzipping mildly gas-phase oxidized MWNTs sonochemically in an organic solvent [22]. Thus far, GNRs from unzipped CNTs have lacked the alignment and ordering on substrates required for device integration.

Here, we show that highly aligned, narrow GNRs can be made from an aligned array of single-walled carbon nanotubes (SWNTs) by our polymer-protected plasma etching method (Figs. 1(a) and 1(b)). Aligned CNTs were first partially embedded in a polymer film and the unprotected sides of CNTs were then exposed to Ar plasma. The polymer film served as an etching mask and enabled the longitudinal unzipping of CNTs [16]. The alignment of CNTs was maintained through embedding in the polymer film, unzipping by plasma and transferring to another substrate. Considering the topographic similarity between narrow GNRs and SWNTs, we performed careful atomic force microscope (AFM) imaging and Raman spectroscopic measurements on the same CNTs before and after the unzipping process to obtain spectroscopic evidence of successful unzipping of CNTs into GNRs. Both the obvious decrease in topographic height obtained by AFM and changes in Raman spectra of the same CNTs

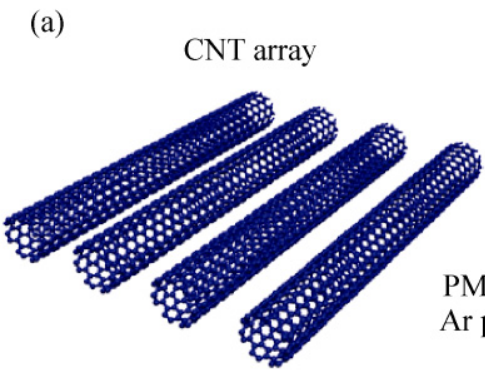

PMMA-protected Ar plasma etching

(b)
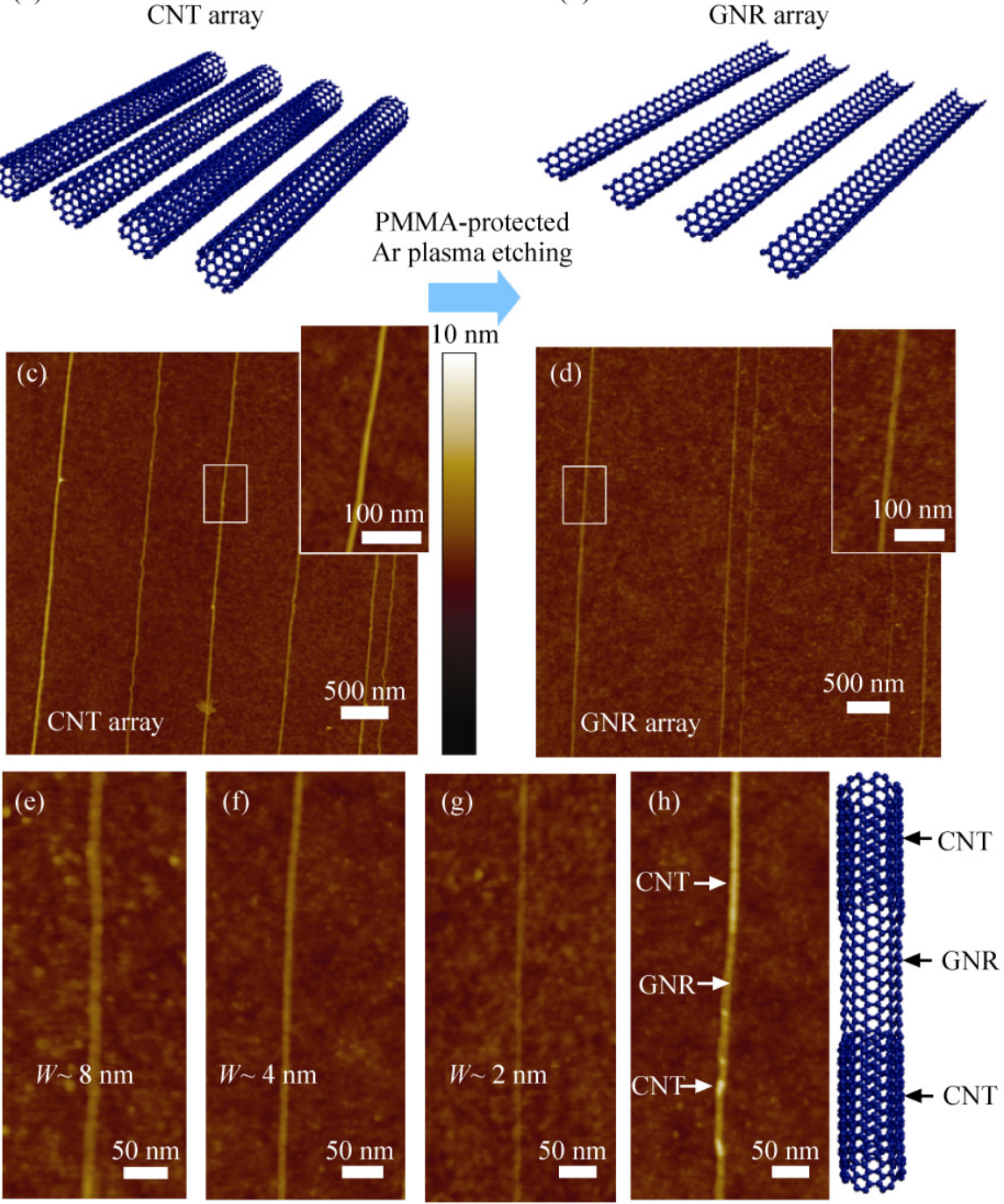

Figure 1 GNR array made from CNT array. Schematic illustration of the fabrication of a GNR array (b) from a CNT array (a) by PMMA-protected plasma etching. Typical AFM images of (c) a pristine CNT array and (d) the resulting GNR array. Insets in (c) and (d) are zoom-in images of an individual CNT and GNR, respectively. AFM images of individual GNRs with widths of (e) $8 \mathrm{~nm}$, (f) $4 \mathrm{~nm}$, and (g) $2 \mathrm{~nm}$. (h) AFM image and schematic illustration of CNT-GNR junctions in a partially unzipped tube 
after unzipping confirmed the creation of GNRs. FETs with an individual GNR and arrays of GNRs as channel materials showed on/off current $\left(I_{\text {on }} / I_{\text {off }}\right)$ ratios up to $10^{3}$ and 20, respectively. Our method also allowed for the formation of crossbars of GNR-GNR and GNR-CNT for the first time.

The starting materials used were dense (1-2 tubes/ $\mu \mathrm{m})$, uniform, highly aligned arrays of long $(\sim 1 \mathrm{~mm})$ CNTs (diameter: $0.6-3.4 \mathrm{~nm} ; 80 \%$ were SWNTs and $\sim 20 \%$ were few-walled CNTs and bundles) grown on ST-cut quartz by CVD [26]. We used markers to track specific CNTs through the unzipping process. We first transferred an as-grown CNT array from quartz to $\mathrm{SiO}_{2} / \mathrm{Si}$ substrates with a sputtered gold marker array by a nano-transfer printing technique [27]. After that, the CNT array was unzipped following a similar procedure to that in our previous work [16]. Briefly, a thin film of poly(methyl methacrylate) (PMMA) was spin-coated on top of the CNT array. After baking, CNTs and gold markers were embedded in the PMMA film. The PMMA/CNTs film with gold markers was then peeled off in a $\mathrm{KOH}$ solution. The film was then exposed to $5 \mathrm{~W}$ Ar plasma for various times. With the protection of PMMA, only the exposed part of the tube sidewall was etched by the Ar plasma. After etching, the film was contact-printed to another $\mathrm{SiO}_{2} / \mathrm{Si}$ substrate. Finally, the PMMA film was removed by acetone vapor and followed by calcination at $300{ }^{\circ} \mathrm{C}$ for $20 \mathrm{~min}$, leaving the unzipped GNRs and gold markers on the target substrate. The protection of PMMA afforded longitudinal unzipping of CNTs and maintained the alignment of CNTs throughout the whole process; both are critical to the creation of highly aligned GNR arrays.

AFM characterization found that the resulting GNRs were mostly single-layered with average height of $\sim 1.0 \mathrm{~nm}$ (Fig. 1(d) and Fig. S-1(b) in the Electronic Supplementary Material (ESM)). The starting CNTs exhibited an average diameter of $\sim 1.4 \mathrm{~nm}$ (Fig. 1(c) and Fig. S-1(a) in the ESM). The GNR array maintained the main features of their parent CNT array including high density, good alignment and ultra-long length. The widths of most obtained GNRs fell into the range of $2-8 \mathrm{~nm}$ (see Fig. S-1(c) in the ESM). As shown in the AFM images (Figs. $1(\mathrm{e})-1(\mathrm{~g})$ and Fig. S-2 in the ESM), the edges of the GNRs appeared smooth and uniform along their length. GNR-CNT junction-like structures were also observed in some partially unzipped tubes due to nonuniform etching caused by local deformations of the PMMA film (Fig. 1(h)). The etching conditions were critical to the successful unzipping of SWNTs. We found that the optimized etching condition was $5 \mathrm{~W}$ (the lowest stable power we could use) for $10 \mathrm{~s}$, which was able to unzip the majority of tubes without introducing many breaks.

By locating specific CNTs with the aid of markers, we obtained AFM images and Raman spectra of the same CNTs before and after plasma etching. As shown in Figs. 2(a) and 2(b), the height of CNTs decreased by $\sim 0.4-0.9 \mathrm{~nm}$ after the unzipping process, which indicated that the CNTs were opened up into GNRs by the Ar plasma. We found that the unzipping efficiency was dependent on the diameter of the parent tubes. Small SWNTs (diameter $<1.0 \mathrm{~nm}$ ) were totally etched by the plasma whilst CNTs with a diameter of $>2.5 \mathrm{~nm}$ were not unzipped under the condition used. All the CNTs with medium diameters $(1.0-2.5 \mathrm{~nm})$ were successfully converted into sub-10 nm GNRs. Therefore, the yield of GNRs ( $\sim 80 \%$ ) was limited by the diameter distribution of the starting CNTs. Besides the decrease in height, important changes were also observed in the Raman spectra of CNTs after unzipping. The Raman spectra of pristine CNTs showed a strong G-band and no obvious D-band, which indicated the high quality of the starting materials [28]. After the unzipping process, the Raman G-band intensity decreased by $\sim 60 \%$ and a strong D-band appeared (Figs. 2(c) to 2(f)). The appearance of the D-band can be attributed to the creation of edges that act as defects responsible for momentum conservation in the double resonance Raman process $[29,30]$. The D-band to G-band $\left(I_{\mathrm{D}} / I_{\mathrm{G}}\right)$ ratio of the GNRs in the array was $\sim 1.5$, much higher than for the parent raw tubes $(<0.01)$. The marked increase in $I_{\mathrm{D}} / I_{\mathrm{G}}$ ratio also confirmed that the CNTs were unzipped by our method. We also performed Raman mapping over individual GNRs (see Fig. S-3 in the ESM) and the averaged $I_{\mathrm{D}} / I_{\mathrm{G}}$ ratio of individual GNRs was $\sim 1.5$. The averaged $I_{\mathrm{D}} / I_{\mathrm{G}}$ ratio of the sub$10 \mathrm{~nm}$ GNRs obtained here was higher than for wider GNRs made by unzipping MWNTs by the same approach due to the much narrower width $(2-8 \mathrm{~nm})$ and proportionally higher defect density at the edges [16]. 

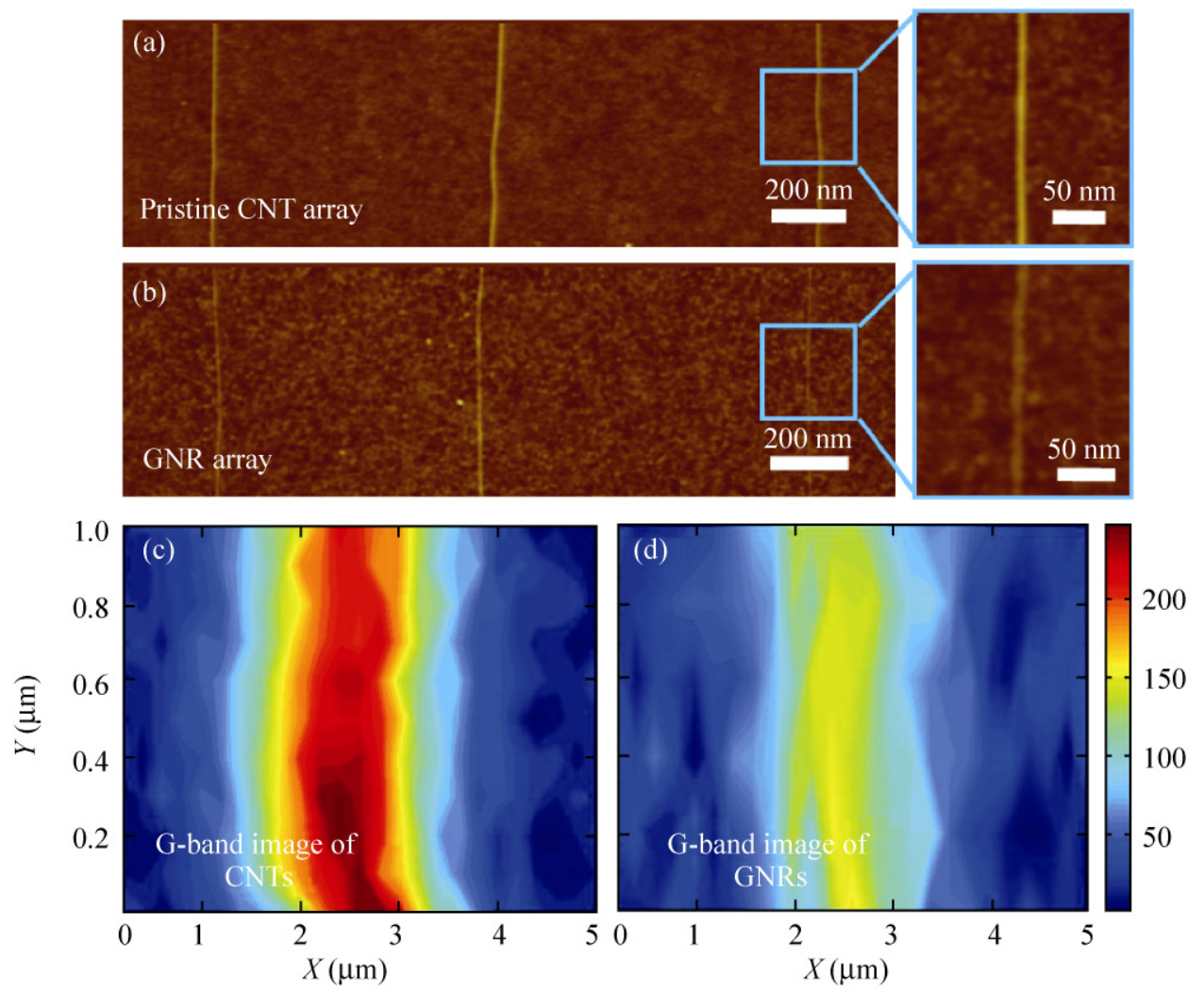

(e)

(f)
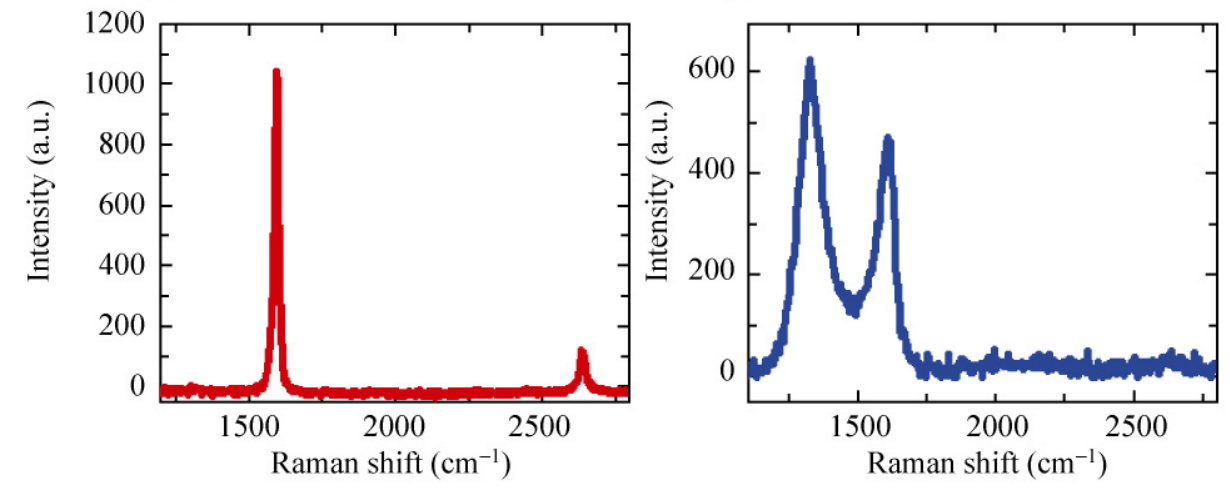

Figure 2 Characterization of the same CNTs before and after unzipping. AFM images of the same CNT array (a) before and (b) after the unzipping process. Insets: zoom-in AFM images of the same CNT before and after unzipping. The height decreases of the three tubes shown in (a) after unzipping were $0.7 \mathrm{~nm}, 0.4 \mathrm{~nm}$, and $0.9 \mathrm{~nm}$ from left to right. (c) and (d) Raman G-band images of the same CNTs shown in (a) and (b), respectively. The intensity of the G-band decreased significantly after the unzipping process. (e) and (f) Averaged Raman spectra of the same CNTs shown in (a) and (b), respectively

We fabricated FETs on an individual GNR and arrays of GNRs, with palladium (Pd) as source/drain (S/D) metal contacts (channel length $L \sim 100 \mathrm{~nm}$ ), a p++ Si backgate, and $100 \mathrm{~nm} \mathrm{SiO}_{2}$ as gate dielectric. The resulting devices of as-made single GNRs showed higher resistance and lower $I_{\text {on }} / I_{\text {off }}$ ratio $\left(<10^{2}\right)$ at room temperature than observed for sub-10 nm GNRs made by the previous sonochemical approach [1], which suggested a high defect density in our as-made GNRs. To improve the quality, we carried out thermal annealing aimed at reducing the number of defects on the GNRs generated by the plasma etching process. We found that thermal annealing in 1 Torr of $\mathrm{H}_{2}$ at $800{ }^{\circ} \mathrm{C}$ for $20 \mathrm{~min}$ improved the electrical properties of GNRs. The $I_{\text {on }} / I_{\text {off }}$ ratios and on-state conductance improved. Figures 3(a) and 3(b) show a $\sim 2 \mathrm{~nm}$ wide 
annealed GNR with an $I_{\text {on }} / I_{\text {off }}$ ratio of $\sim 500$ and on-state current of $\sim 500 \mathrm{nA}$ at a bias voltage $\left(V_{\mathrm{ds}}\right)$ of $1 \mathrm{~V}$. We also fabricated FET devices on a small array of aligned GNRs. A FET made of three aligned GNRs showed an $I_{\text {on }} / I_{\text {off }}$ ratio of 20 and on-state current of $1.5 \mu \mathrm{A}$ at $V_{\mathrm{ds}}=1 \mathrm{~V}$ (Figs. 3(c) and 3(d)). These results suggested that the sub-10 nm GNRs made by the PMMAprotected plasma unzipping method contained a high defect density, partly due to the ultra-narrow widths of the ribbons. Strategies should be devised to improve the quality of aligned narrow GNRs for practical application in nanoelectronics.

The aligned GNRs were explored as building blocks for constructing complex two-dimensional (2-D) structures. We fabricated crossbar structures potentially useful as logic and memory elements in nanoelectronics [31-33] using GNR arrays. Crossbar arrays of GNRs were fabricated by transferring an aligned GNR array on top of another array with a rotation of $90^{\circ}$. The two layers of GNR arrays formed well ordered square mesh structures as evidenced by AFM and Raman G-band images (Figs. 4(a) and 4(b)). Figure 4(c) shows an AFM image of a GNR-GNR junction. Besides GNR-GNR crossbars, we also made two configurations of GNR-CNT crossbar arrays by transferring GNRs on top of CNTs or CNTs on top of GNRs (Fig. 4(d) and Fig. S-4(a) in the ESM). In the GNR-CNT junction, a $\sim 6 \mathrm{~nm}$ wide single layer GNR was placed on the top of a CNT (diameter $\sim 3.3 \mathrm{~nm}$ ) and induced a radial deformation of $\sim 0.3 \mathrm{~nm}$ on the underneath $\mathrm{CNT}$ at

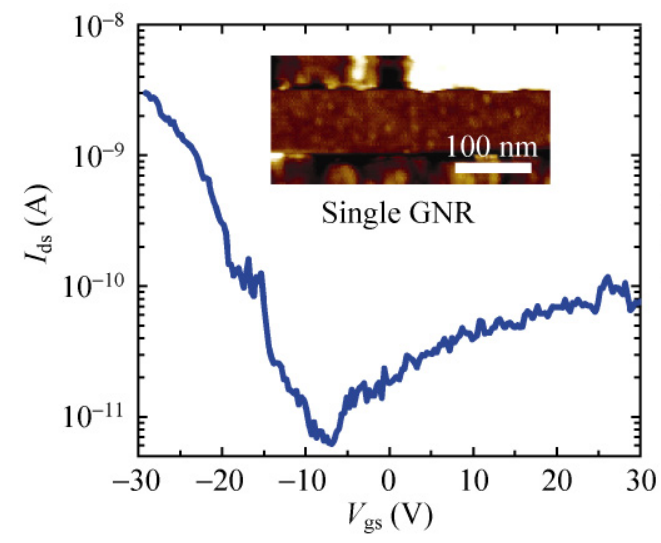

(a)

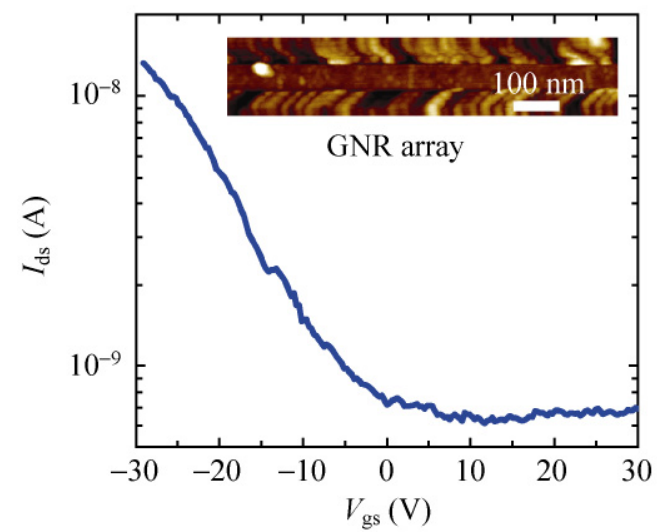

(c)

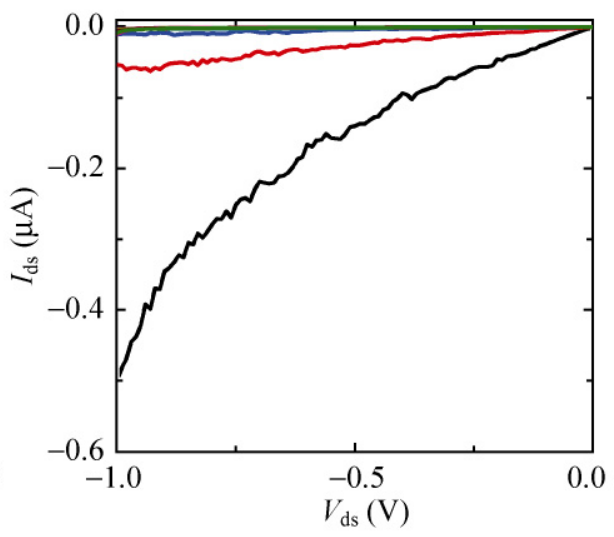

(b)

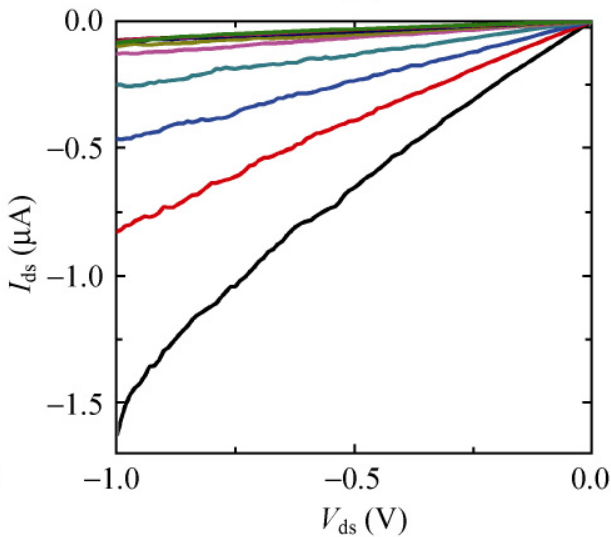

(d)

Figure 3 FETs with an individual GNR and a small array of GNRs. (a) Plot of source-drain current versus gate voltage $\left(I_{\mathrm{ds}}-V_{\mathrm{gs}}\right)$ of a $\sim 2 \mathrm{~nm}$ wide single layer GNR probed in air at $V_{\mathrm{ds}}=10 \mathrm{mV}$. Inset: AFM image of the GNR-FET. The $I_{\mathrm{on}} / I_{\mathrm{off}}$ ratio of this GNR device was $\sim 500$. (b) Current-voltage $\left(I_{\mathrm{ds}}-V_{\mathrm{ds}}\right)$ curves for the device in (a) at various gate biases $V_{\mathrm{gs}}$ from $-30 \mathrm{~V}$ to $30 \mathrm{~V}$ in steps of $5 \mathrm{~V}$ from bottom to top. (c) $I_{\mathrm{ds}}-V_{\mathrm{gs}}$ curve of a FET made on an array of 3 GNRs at $V_{\mathrm{ds}}=10 \mathrm{mV}$. Inset: AFM image of the GNR-FET. The widths of these GNRs were $\sim 5 \mathrm{~nm}, \sim 3 \mathrm{~nm}$, and $\sim 4 \mathrm{~nm}$ from left to right. The $I_{\mathrm{on}} / I_{\mathrm{off}}$ ratio of this device was $\sim 20$. (d) $I_{\mathrm{ds}}-V_{\mathrm{ds}}$ curves for the device in (c) at various gate biases $V_{\mathrm{gs}}$ from $-30 \mathrm{~V}$ to $30 \mathrm{~V}$ in steps of $5 \mathrm{~V}$ from bottom to top 


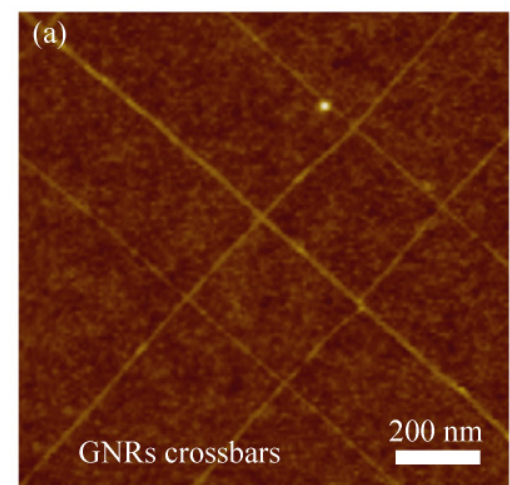

(c)

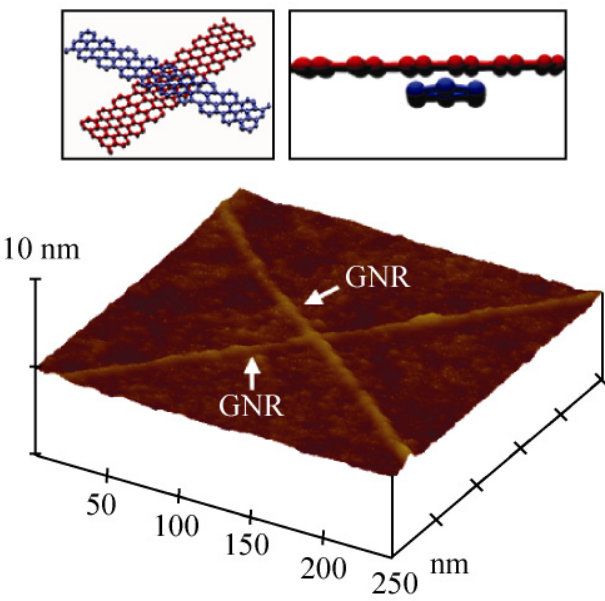

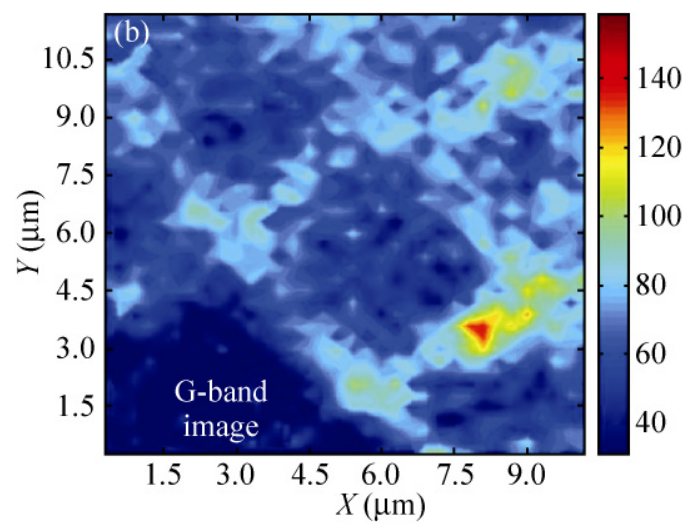

(d)

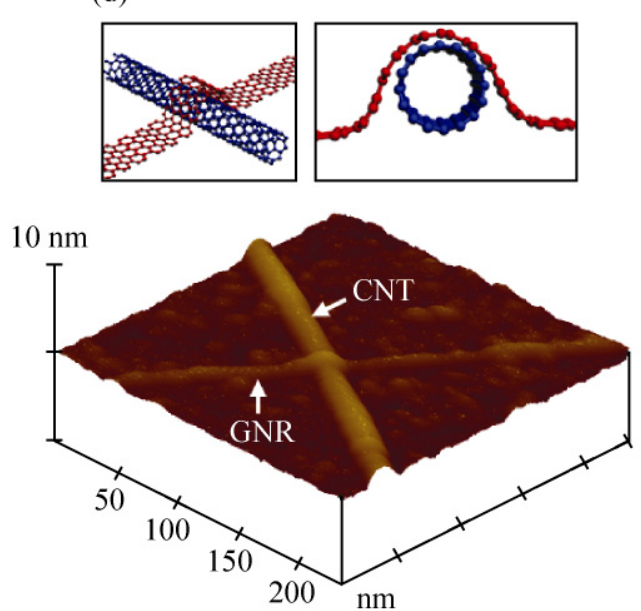

Figure 4 GNR-GNR and GNR-CNT crossbars. (a) AFM image of crossbar array of GNRs. (b) Raman G-band image of the same crossbar array shown in (a). (c) 3-D AFM image and schematic illustration of a GNR-GNR crossbar. The widths of both GNRs were $\sim 5 \mathrm{~nm}$. (d) 3-D AFM image and schematic illustration of a GNR-CNT crossbar. The $\sim 6 \mathrm{~nm}$ wide GNR was on top of a CNT with a diameter of $\sim 3.3 \mathrm{~nm}$. The height of the CNT near the cross point decreased to $\sim 3.0 \mathrm{~nm}$ due to the radial deformation introduced by the GNR (Fig. S-4(c) in the ESM)

the cross point (Fig. 4(d) and Fig. S-4(c) in the ESM), which has also been observed in CNT-CNT junctions [34] (Figs. S-4(b) and S-4(d) in the ESM). No obvious deformation was found when the CNTs were placed on top of GNRs (Fig. S-4(a) in the ESM). The successful fabrication of GNR-GNR and GNR-CNT crossbars will make it possible to explore the fundamental properties and possible applications of these junctions.

In summary, well aligned narrow $(<10 \mathrm{~nm})$ GNR arrays have been made from CNT arrays by polymerprotected plasma unzipping. Well ordered 2-D architectures of GNR-GNR and GNR-CNT crossbars were constructed. Our approach is compatible with semiconductor processing to obtain GNR arrays. However, future work is required to improve the quality of the narrow GNRs unzipped from small nanotubes for applications in high performance nanoelectronics. Nevertheless, the current work represents the beginning of making GNR arrays from nanotube arrays and opens up a way to large scale integration of GNRs into nanoelectronics or optoelectronics.

\section{Experimental}

Preparation of GNR arrays. Aligned CNTs were transferred from a quartz substrate to a $\mathrm{SiO}_{2} / \mathrm{Si}$ substrate with a prefabricated marker array (formed by electron beam lithography (EBL) and sputtering of $30 \mathrm{~nm}$ gold) using the procedures described in Ref. 27. Selected CNTs were located with the aid of markers and characterized using AFM and Raman mapping. Subsequently, a PMMA solution $\left(M_{\mathrm{w}}=495000,5 \%\right.$ in 
anisole) was spin-coated on the substrate at 3000 revolutions per minute (rpm) for $1 \mathrm{~min}$ and then baked at $170{ }^{\circ} \mathrm{C}$ for $2 \mathrm{~h}$ on a hotplate. The PMMA film was peeled off together with the CNTs and gold markers in $1 \mathrm{~mol} / \mathrm{L} \mathrm{KOH}$ solution at $80^{\circ} \mathrm{C}$. Then the film was rinsed with water and dried in air. The film was then exposed to a $5 \mathrm{~W}$ Ar plasma for $10 \mathrm{~s}$ in a plasma reactive ion etching (RIE) system (MRC Model 55) at a base pressure of $40 \mathrm{~m}$ Torr. After etching, the film was printed onto a $\mathrm{SiO}_{2} / \mathrm{Si}$ substrate. PMMA was then removed with the vapor of acetone, leaving the unzipped CNTs and gold markers on the target substrate. Finally, the substrate was calcined at $300{ }^{\circ} \mathrm{C}$ for $20 \mathrm{~min}$ to remove PMMA residue. To improve the quality of GNRs, the calcined GNRs were annealed in $\mathrm{H}_{2}$ at $800{ }^{\circ} \mathrm{C}$ for $20 \mathrm{~min}$ at a pressure of 1 Torr.

Characterization of GNRs. AFM images of the GNR were taken with a Nanoscope IIIa multimode instrument in tapping mode. Raman spectra of GNRs were collected with Horiba Jobin Yvon LabRAM HR Raman microscope with a $633 \mathrm{~nm} \mathrm{He}-\mathrm{Ne}$ laser excitation (spot size $\sim 1 \mu \mathrm{m}$, power $\sim 10 \mathrm{~mW}$ ). The step size of mapping was $100 \mathrm{~nm}$ and the integration time was $10 \mathrm{~s}$ at each spot.

Device fabrication. Before making devices, we first patterned the dense and long GNR arrays within isolated regions using EBL and $\mathrm{O}_{2}$ plasma etching. We then did a second EBL followed by electron beam deposition of $\mathrm{Pd}(30 \mathrm{~nm})$ and lift-off to fabricate arrays of source- and drain-electrodes on the GNRs. The channel length of these devices was $\sim 200 \mathrm{~nm}$ and the width of source and drain electrodes varied from 500 $\mathrm{nm}$ and $5 \mu \mathrm{m}$ for single- and multiple ribbon devices, respectively. The devices were annealed in $\mathrm{Ar}$ at $220^{\circ} \mathrm{C}$ for $15 \mathrm{~min}$ to improve the contact quality. AFM was then used to identify devices with a single or multiple GNR connection. Electrical characterization of the devices was carried out in air using a semiconductor analyzer (Agilent 4156C).

\section{Acknowledgements}

This work was supported by MARCO-MSD, Intel, ONR and graphene-MURI.
Electronic Supplementary Material: Further characterization of aligned GNR arrays and crossbars of GNR-CNT and CNT-CNT by AFM and Raman spectroscopy (four figures) are available in the online version of this article at http://dx.doi.org/10.1007/ s12274-010-1043-z and are accessible free of charge.

Open Access: This article is distributed under the terms of the Creative Commons Attribution Noncommercial License which permits any noncommercial use, distribution, and reproduction in any medium, provided the original author(s) and source are credited.

\section{Reference}

[1] Li, X. L.; Wang, X. R.; Zhang, L.; Lee, S. W.; Dai, H. J. Chemically derived, ultrasmooth graphene nanoribbon semiconductors. Science 2008, 319, 1229-1232.

[2] Wang, X. R.; Ouyang, Y. J.; Li, X. L.; Wang, H. L.; Guo, J.; Dai, H. J. Room-temperature all-semiconducting sub-10-nm graphene nanoribbon field-effect transistors. Phys. Rev. Lett. 2008, 100, 206803.

[3] Wang, X. R.; Li, X. L.; Zhang, L.; Yoon, Y.; Weber, P. K.; Wang, H. L.; Guo, J.; Dai, H. J. N-doping of graphene through electrothermal reactions with ammonia. Science 2009, 324, 768-771.

[4] Cresti, A.; Nemec, N.; Biel, B.; Niebler, G.; Triozon, F.; Cuniberti, G.; Roche, S. Charge transport in disordered graphene-based low dimensional materials. Nano Res. 2008, 1, 361-394.

[5] Chen, Z. H.; Lin, Y. M.; Rooks, M. J.; Avouris, P. Graphene nano-ribbon electronics. Physica E 2007, 40, 228-232.

[6] Han, M. Y.; Ozyilmaz, B.; Zhang, Y. B.; Kim, P. Energy band-gap engineering of graphene nanoribbons. Phys. Rev. Lett. 2007, 98, 206805.

[7] Tapaszto, L.; Dobrik, G.; Lambin, P.; Biro, L. P. Tailoring the atomic structure of graphene nanoribbons by scanning tunnelling microscope lithography. Nat. Nanotechnol. 2008, 3, 397-401.

[8] Bai, J. W.; Duan, X. F.; Huang, Y. Rational fabrication of graphene nanoribbons using a nanowire etch mask. Nano Lett. 2009, 9, 2083-2087.

[9] Wang, X. R.; Dai, H. J. Etching and narrowing of graphene from the edges. Nat. Chem., In press.

[10] Wu, Z. S.; Ren, W. C.; Gao, L. B.; Liu, B. L.; Zhao, J. P.; Cheng, H. M. Efficient synthesis of graphene nanoribbons sonochemically cut from graphene sheets. Nano Res. 2010, $3,16-22$. 
[11] Campos-Delgado, J.; Romo-Herrera, J. M.; Jia, X. T.; Cullen, D. A.; Muramatsu, H.; Kim, Y. A.; Hayashi, T.; Ren, Z. F.; Smith, D. J.; Okuno, Y.; Ohba, T.; Kanoh, H.; Kaneko, K.; Endo, M.; Terrones, H.; Dresselhaus, M. S.; Terrones, M. Bulk production of a new form of $\mathrm{sp}^{2}$ carbon: Crystalline graphene nanoribbons. Nano Lett. 2008, 8, 2773-2778.

[12] Wei, D. C.; Liu, Y. Q.; Zhang, H. L.; Huang, L. P.; Wu, B.; Chen, J. Y.; Yu, G. Scalable synthesis of few-layer graphene ribbons with controlled morphologies by a template method and their applications in nanoelectromechanical switches. $J$. Am. Chem. Soc. 2009, 131, 11147-11154.

[13] Datta, S. S.; Strachan, D. R.; Khamis, S. M.; Johnson, A. T. C. Crystallographic etching of few-layer graphene. Nano Lett. 2008, 8, 1912-1915.

[14] Ci, L. J.; Xu, Z. P.; Wang, L. L.; Gao, W.; Ding, F.; Kelly, K. F.; Yakobson, B. I.; Ajayan, P. M. Controlled nanocutting of graphene. Nano Res. 2008, 1, 116-122.

[15] Campos, L. C.; Manfrinato, V. R.; Sanchez-Yamagishi, J. D.; Kong, J.; Jarillo-Herrero, P. Anisotropic etching and nanoribbon formation in single-layer graphene. Nano Lett. 2009, 9, 2600-2604.

[16] Jiao, L. Y.; Zhang, L.; Wang, X. R.; Diankov, G.; Dai, H. J. Narrow graphene nanoribbons from carbon nanotubes. Nature 2009, 458, 877-880.

[17] Kosynkin, D. V.; Higginbotham, A. L.; Sinitskii, A.; Lomeda, J. R.; Dimiev, A.; Price, B. K.; Tour, J. M. Longitudinal unzipping of carbon nanotubes to form graphene nanoribbons. Nature 2009, 458, 872-876.

[18] Zhang, Z. X.; Sun, Z. Z.; Yao, J.; Kosynkin, D. V.; Tour, J. M. Transforming carbon nanotube devices into nanoribbon devices. J. Am. Chem. Soc. 2009, 131, 13460-13463.

[19] Cano-Marquez, A. G.; Rodriguez-Macias, F. J.; CamposDelgado, J.; Espinosa-Gonzalez, C. G.; Tristan-Lopez, F.; Ramirez-Gonzalez, D.; Cullen, D. A.; Smith, D. J.; Terrones, M.; Vega-Cantu, Y. I. Ex-MWNTs: Graphene sheets and ribbons produced by lithium intercalation and exfoliation of carbon nanotubes. Nano Lett. 2009, 9, 1527-1533.

[20] Elias, A. L.; Botello-Mendez, A. R.; Meneses-Rodriguez, D.; Gonzalez, V. J.; Ramirez-Gonzalez, D.; Ci, L. J.; MunozSandoval, E.; Ajayan, P. M.; Terrones, H.; Terrones, M. Longitudinal cutting of pure and doped carbon nanotubes to form graphitic nanoribbons using metal clusters as nanoscalpels. Nano Lett. 2010, 10, 366-372.

[21] Kim, W. S.; Moon, S. Y.; Bang, S. Y.; Choi, B. G.; Ham, H.; Sekino, T.; Shim, K. B. Fabrication of graphene layers from multiwalled carbon nanotubes using high dc pulse. Appl. Phys. Lett. 2009, 95, 083103.

[22] Jiao, L. Y.; Wang, X. R.; Diankov, G.; Wang, H. L.; Dai, H. J. Facile synthesis of high-quality graphene nanoribbons. Nat. Nanotechnol. [Online early access]. DOI: 10.1038/nnano.2010.54.

[23] Dai, H. J. Carbon nanotubes: Opportunities and challenges. Surf. Sci. 2002, 500, 218-241.

[24] Jorio, A.; Dresselhaus, G.; Dresselhaus, M. S. Carbon Nanotubes: Advanced Topics in the Synthesis, Structure, Properties and Applications; Springer-Verlag: Berlin, 2008.

[25] Zhou, W. W.; Ding, L.; Liu, J. Role of catalysts in the surface synthesis of single-walled carbon nanotubes. Nano Res. 2009, 2, 593-598.

[26] Ding, L.; Yuan, D. N.; Liu, J. Growth of high-density parallel arrays of long single-walled carbon nanotubes on quartz substrates. J. Am. Chem. Soc. 2008, 130, 5428-5429.

[27] Jiao, L. Y.; Fan, B.; Xian, X. J.; Wu, Z. Y.; Zhang, J.; Liu, Z. F. Creation of nanostructures with poly(methyl methacrylate)mediated nanotransfer printing. J. Am. Chem. Soc. 2008, 130, 12612-12613.

[28] Dresselhaus, M. S.; Dresselhaus, G.; Saito, R.; Jorio, A. Raman spectroscopy of carbon nanotubes. Phys. Rep. 2005, 409, 47-99.

[29] Cancado, L.G.; Pimenta, M. A.; Neves, B. R. A.; Dantas, M. S. S; Jorio, A. Influence of the atomic structure on the Raman spectra of graphite edges. Phys. Rev. Lett. 2004, 93, 247401.

[30] Casiraghi, C.; Hartschuh, A.; Qian, H.; Piscanec, S.; Georgi, C.; Fasoli, A.; Novoselov, K. S.; Basko, D. M.; Ferrari, A. C. Raman spectroscopy of graphene edges. Nano Lett. 2009, 9, 1433-1441.

[31] Rueckes, T.; Kim, K.; Joselevich, E.; Tseng, G. Y.; Cheung, C. L.; Lieber, C. M. Carbon nanotube-based nonvolatile random access memory for molecular computing. Science 2000, 289, 94-97.

[32] Melosh, N. A.; Boukai, A.; Diana, F.; Gerardot, B.; Badolato, A.; Petroff, P. M.; Heath, J. R. Ultrahigh-density nanowire lattices and circuits. Science 2003, 300, 112-115.

[33] Fuhrer, M. S.; Nygard, J.; Shih, L.; Forero, M.; Yoon, Y. G.; Mazzoni, M. S. C.; Choi, H. J.; Ihm, J.; Louie, S. G.; Zettl, A.; McEuen, P. L. Crossed nanotube junctions. Science 2000, 288, 494-497.

[34] Ismach, A.; Joselevich, E. Orthogonal self-assembly of carbon nanotube crossbar architectures by simultaneous graphoepitaxy and field-directed growth. Nano Lett. 2006, 6, 1706-1710. 